\title{
„ICH KANN JA NICHT AN DIE FH KOMMEN UND BRAUCHE DAS WIRKLICH GANZ DRINGEND ...“. MÖGLICHKEITEN EINER FACHHOCHSCHULBIBLIOTHEK EFFIZIENT BENUTZER*INNENWÜNSCHE IN ZEITEN DER COVID-19 KRISE ZU ERFÜLLEN
}

\section{von Andreas Hepperger und Elisabeth Gstöttenmeier}

Zusammenfassung: Vieles, das wir heute in den Medien über Krankheitswellen, Schulschließungen oder unmittelbar bevorstehende Impfungen lesen, erinnert augenscheinlich an den Verlauf der Influenza-Pandemie von 1918-1920. Ein wesentlicher Unterschied zu heute ist jedoch die zur Verfügung stehende elektronische Infrastruktur, die uns erlaubt ortsungebunden Studierende und Lehrende mit bibliothekarischen Dienstleistungen zu versorgen. In diesem Beitrag wird dargestellt, wie die Bibliothek der FH Burgenland mit bereits bestehenden Mitteln (Alma-Summon, Microsoft Teams) sowie dem Einsatz zusätzlicher Tools wie LibAnswers den abrupten Einbruch der physischen Benutzung (inkl. Dienstleistungen vor Ort) kompensieren konnte und diese Lösungen auch zukünftig verwenden wird. Schließlich wird gezeigt, wie mit einfachen Mitteln (Microsoft Office) ein Dashboard zur Auslastung der Sitzplätze in der Bibliothek umgesetzt und bereits anderen FH Bibliotheken zur Weiternutzung zur Verfügung gestellt werden konnte.

Schlagwörter: Fachhochschule; Bibliothek; COVID-19; Benutzung

\section{“AS I CANNOT COME TO THE UNIVERSITY, HOW CAN I GET THE BOOK, THAT I REALLY NEED RIGHT AWAY...”. OPPORTUNITIES FOR A UNIVERSITY LIBRARY (FH) TO EFFICIENTLY MEET USER REQUESTS IN TIMES OF THE COVID-19 CRISIS}

Abstract: A lot of what we read today in the media about coronavirus waves, school closings, and soon available vaccines reminds of the influenza pandemic 19181920. An essential difference is the electronic infrastructure that allows us to supply users with online library services. This paper illustrates how the library of the University of Applied Sciences Burgenland managed to compensate the sudden decrease of physical borrowing with already available digital tools (Alma-Summon, Microsoft Teams), and new ones like LibAnswers. Finally, it will be shown how by simple means (Microsoft Office) a library seats availability dashboard was realized and shared with other libraries. 
Keywords: University of Applied Sciences; Library; COVID-19; Readers' Services

DOI: https://doi.org/10.31263/voebm.v73i3-4.5331

(C) Andreas Hepperger, Elisabeth Gstöttenmeier

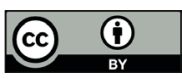

Dieses Werk ist - exkl. einzelner Logos und Abbildungen - lizenziert unter einer Creative-Commons-Lizenz Namensnennung 4.0 International-Lizenz

\section{Einleitung}

Als im März 2020 die ersten Ausgangsbeschränkungen in Kraft getreten sind und sich damit auch abzeichnete, dass die Bibliotheken der FH Burgenland in Eisenstadt und Pinkafeld vorübergehend für die physische Benutzung geschlossen werden müssen, war dies für uns natürlich eine vollkommen neuartige Situation, auf die wir - wie so viele andere - möglichst kurzfristig reagieren mussten. Ein Blick in die Ausleihstatistik Jänner bis Oktober 2020 zeigt, wie drastisch die Anzahl der Entlehnungen in diesem Zeitraum eingebrochen ist.

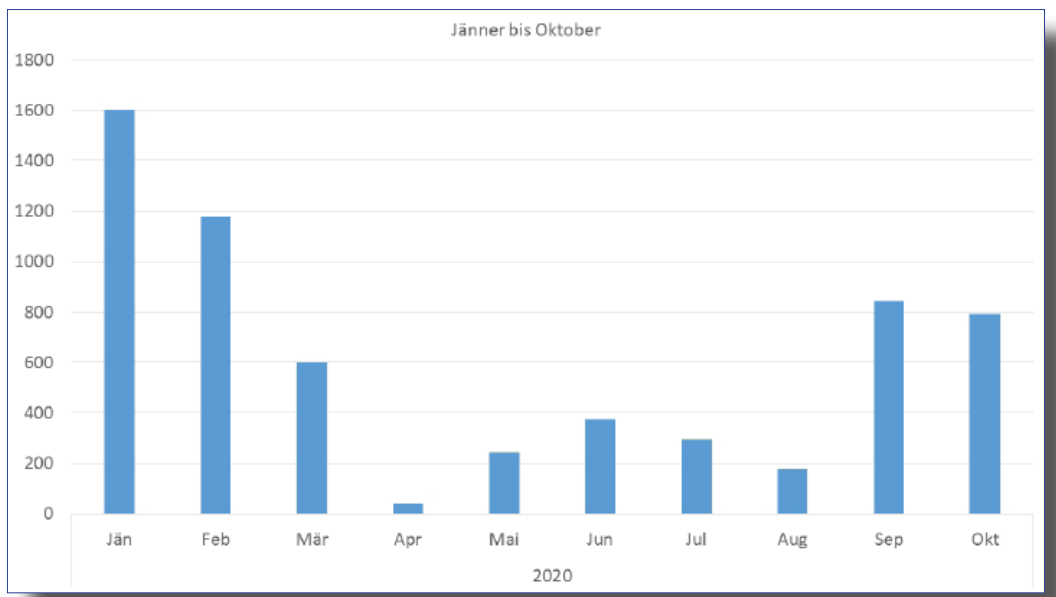

Abb. 1: Ausleihstatistik der Bibliotheken der FH Burgenland in Eisenstadt und Pinkafeld von Jänner bis Oktober 2020

Historisch gesehen sind vergleichbare Situationen vor nicht allzu langer Zeit zu finden. So brauchen wir nur ca. 100 Jahre zurück auf die Influenza-Pandemie von 1918-1920 zu blicken, bei der damals beispielsweise im Oktober 1918 sämtliche Schulen in Ungarn geschlossen wurden. 


\section{Die fpanifore Corippe.}

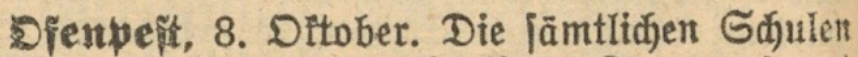
in $1 \mathrm{ng}$ arn fino am heutigen Iage gejpert worbent, da bie Siranffyeit in Den Derfóiedenten Brovinzitäbten einen geradezu ungebeuerlicăen IImfong annimmt.

Abb. 2: Freie Stimmen, 10. Oktober 1918 (ÖNB, ANNO - Austrian Newspapers Online, http://anno.onb.ac.at/cgi-content/anno?aid=fst \&datum $=19181010 \&$ seite $=2 \& z o o m=33$ )

Der wesentliche Unterschied zu heute besteht aber in dem Umstand, dass wir als Bibliothek nicht mehr nur auf die physische Benutzung angewiesen sind, sondern auf eine umfangreiche elektronische Infrastruktur zurückgreifen können, die es uns erlaubt unsere Studierenden ortsunabhängig mit Literatur zu versorgen. Dies gilt im Besonderen für unsere Institution, die über einen Anteil von $69 \%$ berufsbegleitend Studierenden verfügt.

\section{Adaptierungen im Bereich der elektronischen Benutzung}

In einem ersten Schritt haben wir darauf geachtet, die Ankaufswünsche für Bücher so gut als möglich nur mehr in elektronischer Form zu erfüllen. Für den Beschaffungsprozess hatten wir schon bisher die Möglichkeit des Discovery Service Summon genutzt. Dieses ermöglicht auch das Durchsuchen von Beständen, die nicht von der FH Burgenland lizenziert bzw. angeboten werden. Direkt aus den Suchresultaten kann die benötigte Literatur im Zusammenspiel mit der Bibliotheksplattform Alma bestellt werden. Dieses Service stand bereits allen Studierenden und Lehrenden nach erfolgter Anmeldung im Bibliotheksystem zur Verfügung.

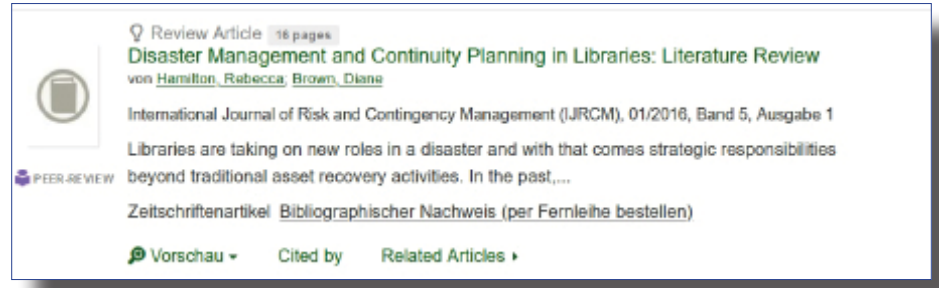

Abb. 3: Beispiel-Datensatz für einen nicht an der FH Burgenland verfügbaren Artikel 
Der Bestell-Workflow war den Benutzer*innen bereits bekannt, da er in den Schulungen der Bibliothek regelmäßig vorgestellt und auch in einem Videotutorial erklärt wird. Zusätzlich haben wir unsere E-Books durch EBS-Angebote (Evidence Based Selection) wie jene von UTB oder Testzugänge wie von De Gruyter, MIT Press o. ä. erweitern können. All diese Inhalte konnten durch die bereits bestehenden Workflows via Alma unseren Benutzer*innen in gewohnter Weise zugänglich gemacht werden und mehr oder weniger einfach in Summon aktiviert bzw. - nach Ablauf der Angebote - wieder deaktiviert werden. Darüber hinaus haben wir auf unserer Webseite auch auf Ressourcen hingewiesen, die von Verlagen vorübergehend frei zur Verfügung gestellt, aber nicht in Alma/Summon integriert werden konnten wie z. B. von Austrian Standards oder spezielle Sammlungen, wie die Coronavirus Research Database von ProQuest.

Natürlich kann dies nicht die individuelle Betreuung vor Ort ersetzen. Deshalb haben wir uns dazu entschlossen die cloudbasierte Anwendung LibAnswers von Springshare zu lizenzieren, diese wird bereits von über 6.000 Bibliotheken weltweit eingesetzt. Für die FH Burgenland waren primär die Funktionalitäten FAQ- und Ticketing-System von Interesse. Nach einer kurzen Testphase stellte sich heraus, dass diese schnell und mit geringem Schulungsaufwand - vom Anbieter wird umfassendes Schulungsmaterial inkl. Videos angeboten - vom Bibliothekspersonal eingesetzt werden kann. Zuerst wurden bereits bestehende FAQs der Webseite übernommen, und mit dem Anstieg an Fragen über das Ticketing-System wurden diese sukzessive ausgewertet und eingearbeitet.

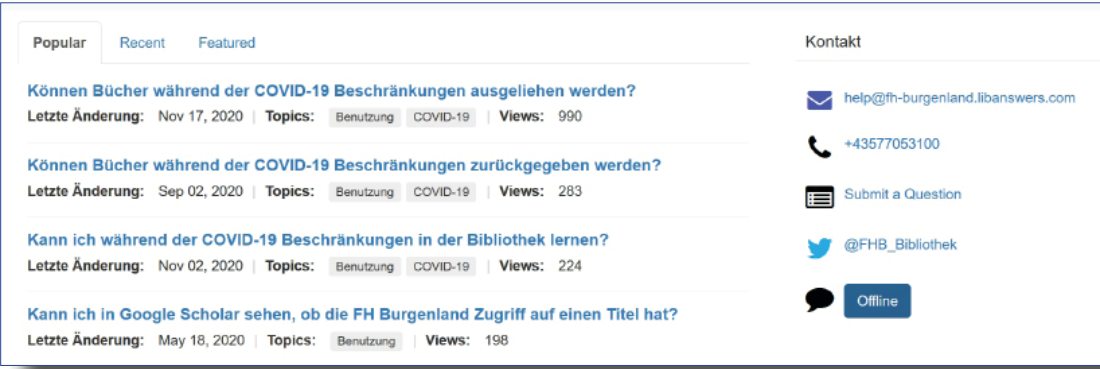

Abb. 4: Bibliotheken der FH Burgenland, LibAnswers (https://fh-burgenland.libanswers.com/)

Nach der Integration der Arbeitsabläufe von LibAnswers in den Arbeitsalltag kam es zu einer raschen Akzeptanz durch die Studierenden. In der Folge haben wir auch die angebotene Chat-Funktion aktiviert und arbeiten gerade daran, diese mit Microsoft Teams zu verknüpfen, damit wir unsere 
Benutzer*innen auch wieder vermehrt individuell und persönlich unterstützen können.

Da wir unsere Suchmaschine als One-Stop-Shop für fast alle bibliothekarischen Dienstleistungen sehen, sind die LibAnswers Funktionalitäten auch in Summon verfügbar. So werden dort die am häufigsten benutzten FAQs automatisch angezeigt und natürlich ist es auch möglich die Chat-Funktion von hier zu starten. Für das Summon-Dezember-Release 2020 wurde darüber hinaus noch eine erweiterte Integration mit LibAnswers angekündigt.

\section{Adaptierungen im Bereich der physischen Benutzung}

Nach Beendigung der Ausgangsbeschränkungen im April 2020 stellten wir an beiden Standorten die Buchrückgabekästen vor den Gebäuden in jeweils überdachten und somit geschützten Bereichen auf, damit nach Terminvereinbarung die Medien in Bibliothekstaschen auf den Kästen in einem Time Slot von 30 Minuten abgeholt werden konnten. Dies ermöglichte uns den Benutzer*innen auch wieder die gewünschten physischen Medien zur Verfügung zu stellen.

Die Terminvergabe gestalteten wir anfänglich mit einem Excel-Sheet, auf das alle Mitarbeiter*innen Zugriff hatten. Seit dem Alma-September-Release 2020 verwendeten wir dafür den Appointment Scheduler aus dem CloudApp-Center, der einfach zu implementieren und leicht anzupassen war.

Für das laufende Wintersemester entschied sich die Geschäftsführung mit dem COVID-19-Krisenstab nach der Leitlinie „So wenig Präsenz wie möglich, so viel wie notwendig, so sicher wie irgendwie machbar" dafür, dass die gesamte Fachhochschule mit der Corona-Ampel-Phase „orange“ startete. Vorrangiges Argument war, dass die Lehrenden, Studierenden und Mitarbeitenden aus verschiedensten Regionen des Landes anreisen und es somit schwierig ist, sich an verschiedene ortsgebundene Ampelschaltungen der Regierung zu halten.

Konkret bedeutete das: am gesamten Campus Maskenpflicht, ca. zwei Drittel der Lehre wurden auf online umgestellt, die Räume wurden entsprechend adaptiert (weniger Sitzplätze, größere Abstände). Für die Bibliotheken entschieden wir uns für eine Gesamtnutzer*innenzahl von 20 Personen für Eisenstadt und vier Personen für Pinkafeld.

Für das Monitoring der Sitzplätze haben wir auf bereits vorhandene Werkzeuge zurückgegriffen. Mit Hilfe von Microsoft Excel und PowerPoint 
haben wir dafür ein Dashboard entwickelt, das auf einem Bildschirm am Eingangsbereich die belegten sowie verfügbaren Arbeitsplätze anzeigt. Dabei werden im Excel-Sheet die verfügbaren Arbeitsplätze von den Bibliotheksmitarbeiter*innen verwaltet und in PowerPoint die Auslastung automatisch angezeigt.

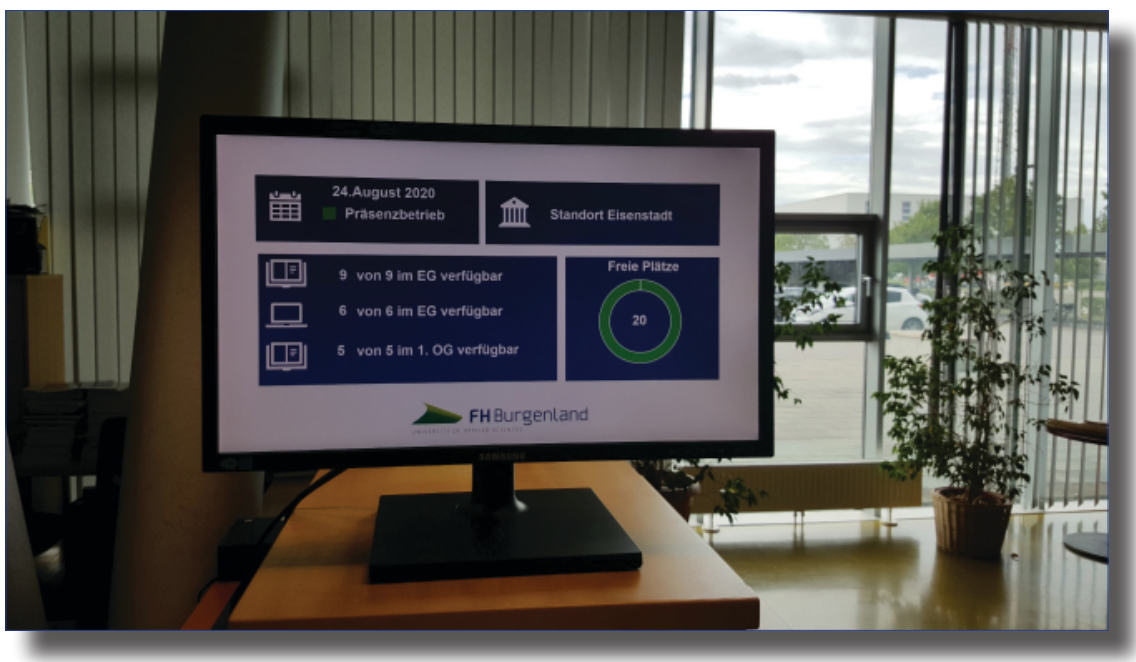

Abb. 5: Dashboard für das Sitzplatz-Monitoring (Foto: Andreas Hepperger)

Diese Lösung konnten wir auch schon anderen Fachhochschulbibliotheken zur Verfügung stellen.

Zu Semesterbeginn standen wir heuer natürlich auch vor der Herausforderung, wie wir für die Studienanfänger*innen die Bibliothekseinführungen gestalten konnten. Aus pragmatischen Gründen führten wir die Online-Schulungen mit den bereits bei Mitarbeiter*innen sowie Benutzer*innen bekannten Tools Microsoft Teams und Cisco Webex durch und verwiesen vermehrt auf unsere Schulungsvideos.

\section{Resümee}

Die letzten Monate waren für uns nicht nur in bibliothekarischer Sicht eine besonders herausfordernde Zeit. Die Mitarbeiter*innen mussten sich innerhalb von kürzester Zeit komplett auf die neuen Gegebenheiten des Homeoffice umstellen und konnten sich in erstaunlich kurzer Zeit mit elektronischen Hilfsmitteln wie Microsoft Teams neu organisieren. So konnte 
die schrittweise Öffnung der physischen bibliothekarischen Services sowie auch die neuerliche Reduktion koordiniert werden. Damit einher ging natürlich auch ein hohes Maß an Flexibilität aller Beteiligten, da sich Öffnungszeiten, Rahmenbedingungen in sehr kurzen Abständen änderten und noch laufend ändern. Eine wesentliche Rolle trägt dazu auch die Hochschulleitung bei, die der Bibliothek ein hohes Maß an Vertrauen und Unterstützung bei den notwendigen Maßnahmen entgegenbrachte und bringt.

Innerhalb der Bibliothek wurde die bereits enge Zusammenarbeit noch intensiviert, und gerade die virtuelle Anbindung des Standortes Pinkafeld wird sicher auch in Zukunft in dieser Form fortgeführt. Erfreulicherweise konnte auch die Verbindung zu allen Akteur*innen des Fachhochschullebens während der letzten Monate auf die ein oder andere Weise immer aufrechterhalten werden. Die Studierenden und Lehrenden wurden weitestgehend ohne Unterbrechung weiter serviciert. Zum einen gelang dies durch eine bereits vorhandene umfangreiche elektronische Infrastruktur und zum anderen durch die Einführung von Tools wie LibAnswers, die gerade sukzessive ausgebaut und auch zukünftig eingesetzt werden.

Mag. Andreas Hepperger, MSc

ORCID iD: https://orcid.org/0000-0002-5091-0112 Fachhochschule Burgenland GmbH, Bibliothek E-Mail: andreas.hepperger@fh-burgenland.at

Mag. ${ }^{a}$ Elisabeth Gstöttenmeier Fachhochschule Burgenland GmbH, Bibliothek Pinkafeld E-Mail: elisabeth.gstoettenmeier@fh-burgenland.at 